\title{
Supporting Visual Assessment of Food and Nutrient Intake in a Clinical Care Setting
}

\author{
Rob Comber ${ }^{1}$, Jack Weeden ${ }^{1}$, Jennifer Hoare ${ }^{2}$, Stephen Lindsay ${ }^{1}$, Gemma Teal ${ }^{3}$, \\ Alastair Macdonald ${ }^{3}$, Lisa Methven ${ }^{4}$, Paula Moynihan ${ }^{2}$ and Patrick Olivier ${ }^{1}$ \\ ${ }^{1}$ Culture Lab, \\ School of Computing \\ ${ }^{2}$ Institute for Ageing and \\ ${ }^{3}$ School of Design, \\ Health, Newcastle \\ University, UK \\ Science, Newcastle \\ University, UK \\ \{robert.comber/ \\ paula.moynihan@ncl.ac. \\ uk \\ Glasgow School of Art, \\ ${ }^{4}$ Department of Food \\ Glasgow, Scotland \\ \{g.teal/a.macdonald\}@gs \\ a.ac.uk \\ and Nutritional Sciences, \\ University of Reading, \\ UK
}

p.1.olivier\}@ncl.ac.uk

\begin{abstract}
Monitoring nutritional intake is an important aspect of the care of older people, particularly for those at risk of malnutrition. Current practice for monitoring food intake relies on hand written food charts that have several inadequacies. We describe the design and validation of a tool for computer-assisted visual assessment of patient food and nutrient intake. To estimate food consumption, the application compares the pixels the user rubbed out against predefined graphical masks. Weight of food consumed is calculated as a percentage of pixels rubbed out against pixels in the mask. Results suggest that the application may be a useful tool for the conservative assessment of nutritional intake in hospitals.
\end{abstract}

\section{Author Keywords}

Nutrition; dietary assessment; portion estimation;

\section{ACM Classification Keywords}

H.5.m [Information interfaces and presentation (e.g., HCI)]: Miscellaneous;

\section{INTRODUCTION}

Monitoring food and nutrient intake is particularly important for those at risk of malnutrition, such as older people in hospitals or care homes. According to the British Association for Parenteral and Enteral Nutrition [10] as many as $33 \%$ of adults are at medium or high risk of malnutrition on admission to hospitals or care homes, and $60 \%$ of older people are at risk [1]. Malnutrition on admission indicates a need for the development of integrated multi-disciplinary strategies to identify, prevent and treat malnutrition across all health care settings. However, hospitalization can lead to further deterioration [e.g. 7] even when additional nutritional supplementation is provided [4]. Therefore, accurate monitoring of regular dietary intake is a key part of patient care.

Permission to make digital or hard copies of all or part of this work for personal or classroom use is granted without fee provided that copies are not made or distributed for profit or commercial advantage and that copies bear this notice and the full citation on the first page. To copy otherwise, or republish, to post on servers or to redistribute to lists, requires prior specific permission and/or a fee.

CHI'12, May 5-10, 2012, Austin, Texas, USA

Copyright 2012 ACM 978-1-4503-1015-4/12/05...\$10.00.

\section{Dietary assessment methodologies}

In the UK the accurate measurement of the food and nutrient intake of individuals in hospitals is not a routine practice. Some patients may have their dietary intake assessed by a dietician but resources limit this to a few highly selected individuals. Nursing staff typically record subjective written assessments of the amount of food consumed as either "none, half or all" on patient food charts. This information is not accurate enough to be useful, as it does not inform on the intake of calories or nutrients. Assessing the amount of food (and subsequently nutrients) consumed from a partially consumed meal constitutes a significant interaction design challenge given the manpower, skill and time required to assess food and nutrient intake in the large number of patients that are at risk of malnutrition. Current methods, which are most suited to the assessment of average intakes by populations include: 1) weighed food intakes where the participant weighs and records foods pre- and post-consumption in a food diary; 2) estimated food diaries, where the participant self-reports estimated amounts of foods consumed in a food diary and 3) food frequency questionnaires [8]. These methods require a trained dietician to translate the information reported into actual intake of nutrients. However, the availability of a trained dietician for this purpose is beyond the capacity of hospital dietetic departments [9]. Consequently, evaluation can occur much after the fact, removing the ability to make timely adjustments for the next meal. There is a clear need for a system to accurately and timeously assess food and nutrient intake that can be used by non-specialist staff.

\section{Related work}

A number of digital tools for portion size and plate waste estimation have been developed. These typically replicate existing methods such as 24 hour recall interviews, using digital food atlases (sequences of images showing varying portion sizes for typical foods), and food models. Such photographic measures have been shown to have high accuracy across a number of age groups and populations for estimation of portion size $[3,5]$ but exhibit large variances at the level of the individual and depending on how the images are presented. Difficulties with photographic 
measures include representing 3D foods, the differences in perception, conceptualization and memory across individuals and demographics of individuals [11].

Examining these effects in low literacy populations, [2] describe the development of a prototype mobile digital portion size estimation tool. The prototype tool uses digital images of various objects (e.g. deck of cards, light bulb) and representations of familiar measures (e.g. weights, hand measures) to assist in the estimation of food portion size and plate waste. Overall, when objects or measures are familiar to the evaluator and match certain food characteristics (i.e. solid, liquid, amorphous), they produce accurate assessments. Although such a tool may increase the availability of a dietary monitoring, particularly selfmonitoring, they remain time consuming, as the patient must identify the portion size and plate waste for each food item in a meal and nutritional intake must be calculated by a trained dietician.

\section{THE MAPPMAL BEDSIDE ASSESSMENT TOOL}

The measurement tool works under the assumption of some constraints established through a number of workshops involving clinicians, dieticians and care staff [6]. Firstly, the environment provides a digital information system at the patient's bedside through which the patient can order food, call for assistance, view menus and so on. The mappmal tool would be integrated with this system. Secondly, food images, food weights and nutritional composition can be determined prior to assessment since hospital meals are of a consistent, standardized size and content. Given that hospital meals are standardized, the mappmal assessment tool assumes set portion sizes at the start of a meal and is used to assess plate waste at the end of the meal, that is the amount of food left after consumption. Third, as trained staff are not always available in hospitals to continuously monitor nutritional intake for all patients, the assessment tool is designed to replace the broad estimates of current methods of food consumption in hospital settings and to give timely feedback for patient care. Given the constraints on trained staff for full assessment of food consumption, the mappmal application must be reliable, fast, accurate and sufficiently easy to use for any member of staff.

To assess portions consumed the user replicates a direct visual assessment of the remaining portion size on a touchenabled device by rubbing away the parts of the meal that have been consumed (See Figure 1). To measure food consumption, the application compares the pixels the user rubbed out from the food image against a series of predefined graphical masks. A mask defines the area and position of individual food items in the original image, identifying each with a unique color. Weight of food consumed is calculated as a percentage of pixels rubbed out against pixels in the mask. Nutritional intake is subsequently derived by reference to food composition tables according to the weight of the rubbed out food. As the assessment utilizes direct observation, no intermediary metaphors, objects or measures, which may be culturally exclusive, are required. By using the tool at time of consumption recall bias may be avoided. The software allows the user to add food to the image, should they rub out too much, by redrawing pixels from the original food image.
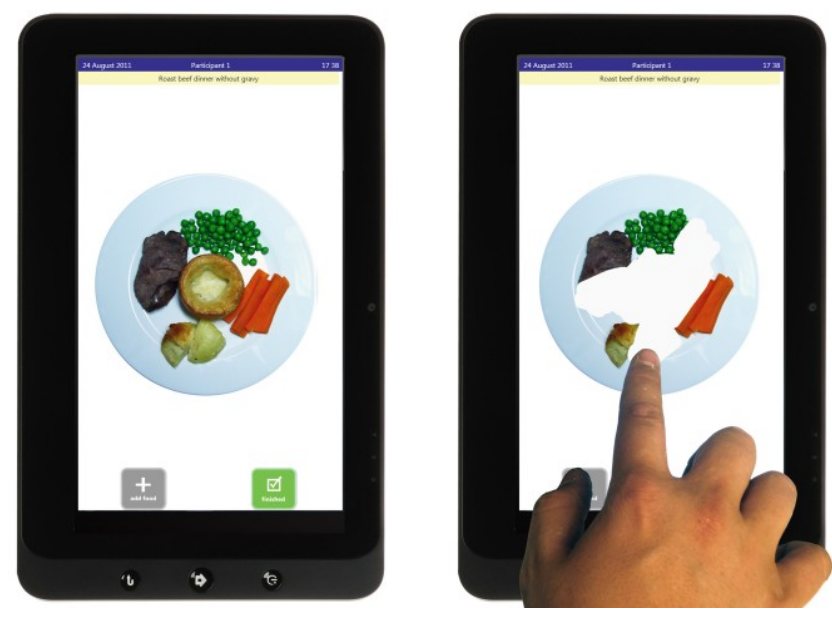

Figure 1: The application prior to and in use. Meals are presented in a top down view. The user rubs out food items based on a visual assessment.

\section{EVALUATION}

A preliminary evaluation of the efficacy of the design was conducted. In this study, we examined: a) how consistent a single user could be, b) how consistent the ratings were between individuals and c) if the system was accurate for novice users. The following describes a typical trial: a meal was prepared and each component weighed using electronic scales. The portion size provided was controlled across trials to reflect those appropriate for hospital patients aged 70 years and over. A volunteer then consumed a portion of each food item from the meal. To ensure that all components could be assessed in the application, part of each component was left uneaten in all trials. The portions of food consumed in each meal were independently rated using the mappmal tool by two primary raters, a system expert and a dietician, and by each of the participants. Participants received no training and were instructed to erase the image to represent the plate in front of them. For each meal a total of 16 evaluations were made $(5 \mathrm{x}$ primary rater $\mathrm{A}, 6 \mathrm{x}$ primary rater $\mathrm{B}$, and $1 \mathrm{x}$ five secondary raters difference in total number of ratings for primary raters due to faster estimations by dietician). Two participants were female, and all had completed a university degree or above. A total of six trials were carried out, representing six different meals with various food types. The primary raters were used to assess intra-rater reliability. When testing inter-rater reliability the primary raters were represented by the average of each of their ratings to avoid being swayed by the number of ratings. Outlying ratings made by participants were excluded. In order to validate the data generated by the application the meal components 
remaining after consumption were manually weighed after the trial.

\section{RESULTS}

Average weights for each food item were calculated and compared with actual weight (See Table 1). On average a subject's use of the application underestimates food consumed by approximately 5.12 grams $(-15.3 \%$ of actual consumed food or $-9.8 \%$ of portion size). Novice secondary raters performed only moderately worse than primary raters ( $7 \mathrm{~g}$ average error as opposed to $5 \mathrm{~g}$ ), though some were

\begin{tabular}{|c|c|c|c|c|}
\hline Meal & Food item & Actual & $\overline{\mathbf{x}}$ & $\boldsymbol{\sigma}$ \\
\hline Beef (with gravy) & Beef & 37 & 23.11 & 5.10 \\
\cline { 2 - 5 } & Yorkshire & 20 & 20.13 & 2.82 \\
\cline { 2 - 5 } & Potato & 18 & 13.11 & 1.99 \\
\cline { 2 - 5 } & Carrots & 25 & 17.15 & 3.41 \\
\cline { 2 - 5 } & Peas & 27 & 24.57 & 2.22 \\
\hline Beef (without gravy) & Beef & 38 & 29.65 & 3.04 \\
\cline { 2 - 5 } & Yorkshire & 20 & 19.26 & 0.61 \\
\cline { 2 - 5 } & Potato & 19 & 14.84 & 1.15 \\
\cline { 2 - 5 } & Carrots & 25 & 17.95 & 2.47 \\
\cline { 2 - 5 } & Peas & 28 & 25.43 & 0.71 \\
\hline Porridge & Porridge & 25 & 23.68 & 4.86 \\
\hline Scram. egg \& toast & Scram. egg & 34 & 24.89 & 8.29 \\
\cline { 2 - 5 } & Toast & 21 & 18.17 & 1.51 \\
\hline Jam sponge \& custard & Custard & 100 & 100.36 & 2.01 \\
\cline { 2 - 5 } & Jam sponge & 77 & 64.69 & 7.03 \\
\hline Fish pie & Carrots & 22 & 16.46 & 3.34 \\
\cline { 2 - 5 } & Fish Pie & 59 & 46.41 & 5.74 \\
\hline
\end{tabular}

Table 1: Actual, mean and standard deviation scores (grams) for each food item.

more accurate). Ratings of individual meals typically took less than 1 minute.

Certain food items presented higher levels of error (E) and standard deviation $(\sigma)$ and were not limited to one food type (i.e. solid, liquid, or amorphous). These include beef (E: $13.89 \mathrm{~g}, \sigma: 5.10 \mathrm{~g}$ ), scrambled egg (E: $-9.11 \mathrm{~g}, \sigma: 8.29 \mathrm{~g}$ ), Jam sponge (E: $-12.31 \mathrm{~g}, \sigma: 7.03 \mathrm{~g}$ ) and fish pie $(\mathrm{E}:-12.59 \mathrm{~g}, \sigma$ : $5.74 \mathrm{~g})$.

Intra-rater reliability was high for primary raters with reliability coefficient of .916 (5 ratings) and $.954(6$ ratings). In both cases, reliability could be increased slightly by excluding the first trial ( $\alpha=.926$ and .964 respectively), suggesting a low learning curve. Inter-rater reliability, including naïve raters, was moderately high, with a reliability coefficient of .856 . Reliability between raters could be increased by the exclusion of the second primary rater $(\alpha=.882)$. This appears to be due to a different rating style used for porridge. Further investigations are required to examine this effect.

\section{DISCUSSION}

The preliminary results suggest that: (1) rating of food consumed was consistent between raters with few exceptions; (2) there was a consistent under-estimation of amount of food consumption for the majority of items; and (3) certain food items, such as fish pie, beef and scrambled egg, were problematic for estimation with higher standard deviations than most. These are discussed in relation to the intended use case.

The consistent rating suggests that both the specific application and the generic visual assessment technique may be reliable tools for estimating food and nutrient intake. The intra-rater reliability shows that a consistent rating is achieved after just one use of the system. This low learning curve will increase the perceived ease of use, which will be important in settings where technology acceptance can be low, such as hospitals. The high interrater reliability may also be important in hospital settings where a number of staff may attend to a patient. Given that the ratings are consistent across raters it would be conceivable that the evaluation of nutritional intake could be carried out by any member of a patient care team. This could contribute to more efficient, effective and timely monitoring of nutrition intake for patients at risk of malnutrition.

However, the assessment appears to be a conservative measure, with most ratings being below the actual weight. [3] report that use of visual estimates typically results in overestimation of portion size. This may remain to be true, as users of the mappmal tool may overestimate food remaining on the plate, thus underestimating food consumed. While this is not ideal for all settings, in a clinical setting underestimation is preferred to overestimation, as the assumption should be made that patients below a target intake are at risk of malnourishment.Difficulties were presented by the nature of consumption of particular food items. In later iterations of the mappmal assessment tool, a variety of measures may be necessary to reflection different food types (i.e. solid, liquid, amorphous). As per [2], known measures such as handfuls, may be more appropriate for amorphous foods, such as fish pie and scrambled egg. The tool may allow individuals to remove known portions, such as a spoon of scrambled egg at a time. In the case of beef, it is likely that the error occurred due to different thickness in beef presented in the image (and therefore assumed in the application) and that on the plate. Therefore, it would be necessary to make secondary assessments using other measures, such as angled images [11] or cross-sectional side views.

Discussions with raters after the measuring exercise suggested that different strategies for estimation were 
employed in the cases of ambiguous foods items. This was most notable for the assessment of porridge where one participant's estimates averaged around $2 \mathrm{~g}$ compared with the $23 \mathrm{~g}$ average for the remainder of the group. The extreme under-estimation appeared to be due to the direct mapping of the visual representation to the bowl. That is, the individual erased only the edges of the porridge that equated to the decrease in height of the porridge. The more accurate raters transformed the approximated consumption and carried out a representative assessment, erasing a continuous block of the porridge corresponding to estimated consumption. This suggests that when appropriate input is ambiguous mental transformations may allow for an accurate estimate. However, this is not ideal since transformations may not be consistent between individuals. Guideline for transformations could be established and relayed at time of use. As with other liquids, a cross-sectional side view may also be effective.

The application could be used to assist in the measurement of food where standardized meals are prepared and where a conservative estimation of nutritional intake might be of benefit. As such, it is ideally situated for use in hospitals where it could provide benefits on a number of levels. The application could prove beneficial for patients, in terms of improved monitoring and timely care, for staff, in terms of decreased effort required to monitor nutrition without decreased accuracy, and for hospital management in the reduction of costs associated with nutritional monitoring. While it is unsuitable to be used by the patients themselves (for reasons of accountability), the simplistic nature of the interface, coupled with the richness of the data it provides, allows a much broader range of staff to assist with the nutritional intake assessments. The general applicability of the approach is limited by the fact that the application requires images of standardized meals to be entered into the system prior to use. Therefore, the application is most suited to environments where meals are planned ahead of time and portion size, quality and composition are relatively standardized, such as the intended use in hospitals where standardized meals are the norm. Further investigation of the tool in a typical use setting is required.

\section{CONCLUSION}

The application provides a reliable but conservative measure of nutritional intake from partially consumed meals. The findings suggest that the application may be suitable for use in a hospital setting where technology acceptance may be low, the demand for efficiency and effectiveness are high, but where the need for accurate monitoring is paramount. The application provides an interaction design that meets these requirements and can contribute to the timely, effective care of patients at risk of malnutrition.

\section{ACKNOWLEDGMENTS}

This research was part of the mappmal project, funded by the New Dynamics of Ageing Programme administrated by the ESRC. We would also like to thank reviewers for their input.

\section{REFERENCES}

1. Age Concern. Hungry to be Heard: The scandal of malnourished older people in hospital. Age Concern England, London, UK, 2006.

2. Chaudry, B., Connelly, K., Siek, K.A., and Welch, J.L. The design of a mobile portion size estimation interface for a low literacy population. In 5 th International Conference on Pervasive Computing Technologies for Healthcare, (2011), 160-167.

3. Hernandez, T., Wilder, L., Kuehan, D., Rubotzky, K., Moser-Veillon, P., Godwin, S., et al., Portion size estimation and expectation of accuracy. J. Food Comp. Anal., 19, (2006), 814-821.

4. Larsson, J., Unosson, M., Ek, A.-C., Nilsson, L., Thorslund, S., and Bjurulf, P. Effect of dietary supplement on nutritional status and clinical outcome in 501 geriatric patients--a randomised study. Clinical Nutrition 9, 4 (1990), 179-184. DOI: 10.1016/02615614(90)90017-M.

5. Lucas, F., Niravong, M., Villeminot, S., Kaaks, R. and Clavel-Chapelon, F. Estimation of food portion size using photographs: validity, strengths, weaknesses and recommendations. Journal of Human Nutrition and Dietetics 8, (1995), 65-74.

6. Macdonald A.S., Teal G., and Moynihan P. A smarter food service for nutritionally vulnerable older adult hospital patients. In Proc. of CWUAAT 10, (2010), 169177.

7. McWhirter, J. P. and Pennington, C. R. Incidence and recognition of malnutrition in hospital. British Medical Journal 308, (1994), 945-948.

8. Moynihan, P., Thomason, J. M., Walls, A. W. G., GrayDonald, K., Morais, J. A., Ghanem, H., Wollin, S., Ellis, J. S., Steele, J., Lund, J., and Feine, J. Researching the impact of oral health on diet and nutritional status: methodological issues. Journal of Dentistry 37, 4 (2009), 237-249.

9. Reilly, H. M. Screening for nutritional risk. Proceedings of the Nutrition Society 55, (1996), 841-853. http://dx.doi.org/10.1017/S0029665196000286

10.Russell, C. and Elia, M. Nutrition Screening Survey in the UK and Republic of Ireland in 2010. A report by the British Association for Parenteral and Enteral Nutrition (BAPEN). BAPEN, Redditch, 2011.

11.Subar, A.F., Crafts, J., Zimmerman, T.P., Wilson, M., Mittl, B., Islam, N.G., et al. Assessment of the accuracy of portion size reports using computer-based food photographs aids in the development of an automated self-administered 24-hour recall. J. Am. Diet. Assoc., 110, (2010), 55-64. 